\title{
The Need for a Mental Health Technology Revolution in the COVID-19 Pandemic
}

\author{
Caroline A. Figueroa ${ }^{1 *}$ and Adrian Aguilera ${ }^{1,2}$ \\ ${ }^{1}$ School of Social Welfare, University of California, Berkeley, Berkeley, CA, United States, 2 Department of Psychiatry, \\ Zuckerberg San Francisco General Hospital, University of California, San Francisco, San Francisco, CA, United States
}

Keywords: mobile health (mHealth), COVID-19, apps and smartphones, telemental health, public mental health, psychological therapies, depression, anxiety

\section{INTRODUCTION}

The current coronavirus 2019 (COVID-19) pandemic not only poses a large threat to the physical health of our population, if we fail to act now, it will also have detrimental long-term consequences for mental health.

Though social distancing is a crucial intervention to slow down the destructive effects of the pandemic, it can lead to isolation, decreased physical activity, and increased rumination, which might particularly hurt those with pre-existing mental illness. Further, the stream of disheartening COVID-19 news provides fodder for increased worry and distress, which can be detrimental for people with anxiety disorders. Early cross-sectional surveys in the United States, Canada, and Europe show an increase in symptoms of depression and anxiety for the general population, associated with COVID-19 concerns (1). Thus, this crisis is exacerbating existing mental health conditions and creating conditions for the development of new ones. Further, if lessons from other outbreaks such as Ebola (2) and SARS (Severe Acute Respiratory Syndrome) (3) are any indication, even after an outbreak is controlled, there will likely be a substantial increase in need for psychological support.

Crucially, this public health crisis will magnify and deepen existing shortcomings of mental health care systems. The US was already facing a mental health crisis before the pandemic: less than half of those with mental illness receive the care that they need (4). Underserved populations, such as low-income or ethnic minority populations, are disproportionately affected; they show the lowest utilization of mental health services (5). Early data suggest that underserved populations pay a larger health toll from COVID-19: they show higher mortality rates $(6,7)$. They are more likely to work in essential jobs putting them at greater risk of contracting COVID-19, and suffer greater economic consequences. All these factors lead to increased stress and anxiety. We will therefore be faced with an even greater relative shortage of trained professionals and means to mental health care during and after this pandemic.

We argue that what we need during a public health crisis like this is a digital mental health revolution: scaling up the delivery of confidential mental health services to patients across a wide range of platforms, from telemental health to mobile interventions such as apps and text messaging. Here, we provide an overview of technological tools which could help to decrease the mental health burden of COVID-19, provide recommendations on how they could be used and scaled-up, and discuss considerations and limitations of mental health technology applications. 


\section{Telehealth}

There is a crucial role for the use of teleconferencing software for therapy sessions during the COVID-19 pandemic. Most studies of teleconferencing services showed that effectiveness is comparable to in-person services across disorders including depression, posttraumatic stress disorder, and anxiety disorders (8). China has had some success with this approach. Researchers recently wrote in a Lancet Commentary that during the worst of the outbreak in January, China successfully provided online psychological counseling and self-help was widely rolled out by mental health professionals in medical institutions, universities, and academic societies (9).

In the US, the pandemic has also catalyzed a rapid adoption of telehealth (10). Medicare now allows for billing for telehealth. Further, the Health Insurance Portability and Accountability Act (HIPAA) has been revisited to permit more medical providers to use HIPAA compliant platforms to communicate with patients. This removes a major barrier to wider adoption of telemedicine and could also provide an outstanding opportunity for patients who previously did not feel comfortable seeking mental health care to now approach these services.

However, it is important to attend to disparities in technology access and digital literacy. Before the pandemic, only one in ten patients in the US used telehealth, and 75\% said that they were unaware of telehealth options or how to access it (11). Recent data from primary care clinics showed that, though video care consults went up by $80 \%$ in late March and early April, minority groups represented a smaller portions of these visits (12). This is partly explained because of a lack of Internet availability, which varies due to limited data plans and lack of $\mathrm{Wi}$ - Fi, and inability to use smartphone features such as downloading apps (13). At the moment, some US telecom providers are offering free Internet services (14). However, longer-term strategies need to be developed to prevent further widening of the digital divide (15), including providing affordable, high speed Internet access, improving usability of telehealth programs, and providing appropriate guidance/training for patients using these services.

\section{Mental Health Smartphone Applications}

Importantly, the use of personal mobile phones presents an opportunity for broad scaling of interventions. Over $90 \%$ of Americans have some type of mobile phone and over $80 \%$ have smartphones (16). Even among low-income Americans (71\%) and older adults (53\%) smartphone ownership is high. Mental health apps have shown effectiveness in decreasing symptoms of depression (17) and anxiety (18). Because of COVID-19, multiple meditation and wellness apps designed by the private sector have now temporarily opened up free memberships to aid in easing anxiety, the majority of these being mindfulness apps (19).

However, there are over 10,000 consumer-available mental health apps in app stores and many of these are not evidencebased (20). Further, though many people download mental health apps, research shows low rates of continued use over longer periods of time (21). It is crucial that mental health providers recommend apps that are backed up by evidence. One helpful resource is Psyberguide (www.psyberguide.org), a non-profit that rates apps based on the strength of the scientific research that supports it, ease of use, and its privacy policies (22). Lastly, in order to improve engagement, providers should follow up with patients on their usage of these apps and integrate the app content into their treatment.

\section{Texting Applications}

In addition to apps, text-messaging platforms could be leveraged to help people cope with mental health challenges evoked by COVID-19. Because texts are also delivered via individuals' devices, they are easy to provide to many at once using automated text-messaging platforms. Text-messaging interventions have demonstrated effectiveness in behavioral health promotion and disease management (23). Importantly, text-messaging is an appropriate tool for low digital literacy populations and underserved groups (24). For instance, our own HIPAA approved texting platform, HealthySMS, was developed with and for low-income populations (mostly Spanish speakers) and shows high acceptability in underserved populations (25). We recently rolled-out a text-messaging study to provide widescale support to interested individuals in the US via daily automated text-messages, containing tips on coping with social distancing and COVID-19 anxiety.

For crisis situations, Crisis Text Line provides free confidential help via text-message. This platform has seen the mention of "coronavirus" in 24\% of conversations from March 30th to April 6th (26). Furthermore, Caremessage, a non-profit organization, has temporarily provided free access to their messaging platform and COVID-19 template text-messaging library with health information (27). In addition, reliable information can also be delivered by health and government organizations automated via text messages. Scaling of information delivery to patients and the public could also relieve health professionals and public health departments, who are already understaffed, underfunded, and overburdened (14).

\section{Social Media}

Social media plays a complicated role in the management of mental health. On the one hand, it can provide positive and supportive connections during a time of physical isolation. Earlier work shows that many people with mental illness are increasingly turning to social media to share their experiences and seek mental health information and advice (28). On the other hand, it can also serve to increase depression and anxiety symptoms based on negative social comparisons and the spread of distressing information (29). For instance, in a recent crosssectional survey of almost 5,000 participants in China, increased social media exposure on COVID-19 was associated with increases in anxiety and depression symptoms (30).

Social media has played a large role in the spread of information since the start of the COVID-19 outbreaks, including misinformation and "fake news". Large social media platforms are now reportedly taking steps to remove false content or conspiracy theories about the pandemic, using 
artificial intelligence (AI); and distribute reliable information, such as developed by the World Health Organization (31).

In China, the government provided online mental health education through popular social media platforms, such as WeChat, Weibo, and TikTok during the height of the outbreak in January (9). In the UK, the National Health Service (NHS) is working with Google, Twitter, Instagram, and Facebook to provide the public with accurate information about COVID19 (32).

Social media also provides a unique opportunity for health professionals to distribute accurate information to their patients and the public, or to highlight available mental health resources. In Wuhan China, mental health professionals uploaded videos of mental health education for the general public through WeChat and other Internet platforms at the early stage of the outbreak (9). In the US and Europe, many physicians have turned to Twitter to share medical information. The social media site has now implemented a mechanism to verify physicians and other scientific experts in an effort to counteract coronavirus misinformation (33).

However, because of the overload of information on social media, misinformation might still spread too fast to be intercepted by AI algorithms $(34,35)$. A recent report of responses from more than 8,000 people from six countries showed that one third reported seeing a significant amount of false or misleading COVID-19 information on social media or messaging platforms (36).

Further, posting information on social media raises the question of how health professionals should respond to the information posted by patients, and how that can impact the therapeutic relationship. Currently, there are no clear guidelines for health professionals, to determine how to act on social media. This calls for a push in quickly establishing such a consensus (37).

\section{DISCUSSION}

The COVID-19 crisis has fast-forwarded the use of technology in mental health care. Technology is crucial in scaling up access to mental health services during and after COVID-19. Given that people interact differently with technology, people of various ages, technical abilities, languages, and levels of literacy will need distinct types of interventions (38).

Older people are particularly vulnerable during this pandemic and already suffer from high rates of loneliness (39). This is strongly associated with greater symptoms of depression and anxiety (40), and physical morbidities and mortality (41). Previous work shows that older adults are interested in using technology to support their mental health, and that mobile health technology is feasible and reliable for assessing cognitive and mental illness (42).

However, older adults and those with low digital literacy might lack prior knowledge of digital technology to fully benefit from these tools (43). Digital health tools suffer from usability issues: they do not always consider digital literacy, health literacy, age, or English proficiency in their design (44). For instance, previous work showed that even the most basic functions of apps are difficult to use for diverse populations (45). Top-funded digital health companies test only $30 \%$ of their apps in people with clinical conditions (46). These factors are important because individuals with lower health literacy have worse health outcomes over time due to difficulty making informed health choices (47).

Therefore, interventions should be specifically targeted toward vulnerable groups, and adapted to their specific needs. This includes design choices such as easy to navigate user interfaces and tailoring vocabulary to older adults or those with low English proficiency (48). Training for individuals with low-tech skills, through outreach programs by healthcare staff may help patients to understand and use digital tools (49). Health systems should prioritize implementation of this crucial service (12).

Further, the right infrastructure needs to be set up to provide digital interventions securely, without personal privacy violations and minimizing the risk of data breaches. Apps and text-messaging must not only be effective, but also safe, secure, and responsible, similar to how therapists are held to standards of responsible practice and confidentiality (42). Therefore, it is imperative that cybersecurity specialists also become involved in ensuring safe technological services (50). Finally, just as they have now shown flexibility with telehealth, insurance companies and health systems should begin covering digital and mobile health interventions.

\section{CONCLUSION}

We are now in the midst of an acute health crisis which calls for a grand upscaling of mental health resources. Technology provides a medium for delivering mental health services remotely and on a wide scale, which is particularly important during social distancing measures. Even when the worst of the COVID-19 pandemic has subsided, it is likely that a large need for mental health support and services delivered through technology will remain. Digital mental health tools should be affordable, accessible, and appropriate for a wide group of individuals with varying ages, languages, and digital literacy. The time to massively invest in high quality and accessible online and mobile mental health in the face of the COVID-19 pandemic, and possible future pandemics, is now.

\section{AUTHOR CONTRIBUTIONS}

CF wrote the first draft of the article. AA contributed to the writing and editing of the manuscript. Both authors contributed to the editing of the final manuscript. 


\section{REFERENCES}

1. Nelson B, Pettitt AK, Flannery J, Allen N. Psychological and Epidemiological Predictors of COVID-19 Concern and Health-Related Behaviors. Psyarxiv (preprint) (2012). doi: 10.31234/osf.io/jftze

2. Mohammed A, Sheikh TL, Poggensee G, Nguku P, Olayinka A, Ohuabunwo $\mathrm{C}$, et al. Mental health in emergency response: lessons from Ebola. Lancet Psychiatry (2015) 2(11):955-7. doi: 10.1016/S2215-0366(15)00451-4

3. Mak IWC, Chu CM, Pan PC, Yiu MGC, Chan VL. Long-term psychiatric morbidities among SARS survivors. Gen Hosp Psychiatry (2009) 31(4):31826. doi: 10.1016/j.genhosppsych.2009.03.001

4. Mental Health America. The state of mental health in America. Author Alexandria, VA: Mental Health America, Inc. (2019).

5. Clancy C, Munier W, Brady J, Moy E, Chaves K, Freeman W, et al. 2012 National Healthcare Quality \& Disparities Report. Rockville, Washington, DC: AHRQ Publications (2013).

6. Kirby T. Evidence mounts on the disproportionate effect of COVID-19 on ethnic minorities. Lancet Respiratory Med (2020). doi: 10.1016/S2213-2600 (20)30228-9

7. Yancy CW. COVID-19 and African Americans. Jama (2020) 323(19):1891-2. doi: $10.1001 /$ jama.2020.6548

8. Ralston AL, Andrews ARIII, Hope DA. Fulfilling the promise of mental health technology to reduce public health disparities: Review and research agenda. Clin Psychol: Sci Practice (2019) 26(1):e12277. doi: 10.1111/cpsp.12277

9. Liu S, Yang L, Zhang C, Xiang Y-T, Liu Z, Hu S, et al. Online mental health services in China during the COVID-19 outbreak. Lancet Psychiatry (2020) 7 (4):e17-e8. doi: 10.1016/S2215-0366(20)30077-8

10. Wosik J, Fudim M, Cameron B, Gellad ZF, Cho A, Phinney D, et al. Telehealth Transformation: COVID-19 and the rise of Virtual Care. J Am Med Inf Assoc: JAMIA (2020) ocaa067. doi: 10.1093/jamia/ocaa067

11. Power JD. U.S. Telehealth Satisfaction Study. SM, J.D. Power (2019).

12. Nouri S, Khoong EC, Lyles CR, Karliner L. Addressing Equity in Telemedicine for Chronic Disease Management During the Covid-19 Pandemic. Catalyst non-issue content. NEJM Catalyst (2020) 1(3).

13. Anderson M, Perrin A, Jiang J. 13\% of Americans don"t use the internet. Who are they. Pew Research Center. (2016). p. 7.

14. New York Times. (2020). [Available from: https://www.usatoday.com/story/ money/2020/03/16/utility-cable-internet-phone-coronavirus-covid-19/ 5060084002/.

15. Yoon H, Jang Y, Vaughan PW, Garcia M. Older adults' Internet use for health information: Digital divide by race/ethnicity and socioeconomic status. J Appl Gerontol (2020) 39(1):105-10. doi: 10.1177/0733464818770772

16. Pew Research Center. Mobile Fact Sheet: Pew Research Center. Pew Research Center: Internet. Science \& Tech. (2018). [Available from: https://www. pewinternet.org/fact-sheet/mobile/.

17. Firth J, Torous J, Nicholas J, Carney R, Pratap A, Rosenbaum S, et al. The efficacy of smartphone-based mental health interventions for depressive symptoms: a meta-analysis of randomized controlled trials. World Psychiatry : Off J World Psychiatr Assoc (WPA) (2017) 16(3):287-98. doi: 10.1002/wps.20472

18. Firth J, Torous J, Nicholas J, Carney R, Rosenbaum S, Sarris J. Can smartphone mental health interventions reduce symptoms of anxiety? A meta-analysis of randomized controlled trials. J Affect Disord (2017) 218:1522. doi: 10.1016/j.jad.2017.04.046

19. Techcrunch. (2020). [Available from: https://techcrunch.com/2020/03/18/ meditation-apps-offer-free-mindfulness-as-social-distancing-takes-a-toll/.

20. Larsen ME, Huckvale K, Nicholas J, Torous J, Birrell L, Li E, et al. Using science to sell apps: evaluation of mental health app store quality claims. NPJ Digit Med (2019) 2(1):1-6. doi: 10.1038/s41746-019-0093-1

21. Baumel A, Muench F, Edan S, Kane JM. Objective user engagement with mental health apps: systematic search and panel-based usage analysis. J Med Internet Res (2019) 21(9):e14567. doi: 10.2196/14567

22. Lipczynska S. Psyberguide: a path through the app jungle. J Ment Health (2019) 28(1):104-. doi: 10.1080/09638237.2017.1417574

23. Berrouiguet S, Baca-García E, Brandt S, Walter M, Courtet P. Fundamentals for future mobile-health (mHealth): a systematic review of mobile phone and web-based text messaging in mental health. J Med Internet Res (2016) 18(6): e135. doi: 10.2196/jmir.5066
24. Pew Research Center. Smartphone Ownership Is Growing Rapidly Around the World, but Not Always Equally. Pew Research Center's Global Attitudes Project (2019).

25. Aguilera A, Berridge C. Qualitative Feedback From a Text Messaging Intervention for Depression: Benefits, Drawbacks, and Cultural Differences. JMIR mHealth uHealth (2014) 2(4):e46. doi: 10.2196/mhealth.3660

26. Crisis Textline. (2020). [Available from: https://www.crisistextline.org/ mental-health/notes-on-coronavirus-how-is-america-feeling-part-4/.

27. CareMessage. (2020). [Available from: https://www.caremessage.org/covid19-response-messaging-safety-net-providers.

28. Naslund JA, Aschbrenner KA, McHugo GJ, Unützer J, Marsch LA, Bartels SJ. Exploring opportunities to support mental health care using social media: A survey of social media users with mental illness. Early Intervent Psychiatry (2019) 13(3):405-13. doi: 10.1111/eip.12496

29. Primack BA, Shensa A, Escobar-Viera CG, Barrett EL, Sidani JE, Colditz JB, et al. Use of multiple social media platforms and symptoms of depression and anxiety: A nationally-representative study among US young adults. Comput Hum Behav (2017) 69:1-9. doi: 10.1016/j.chb.2016.11.013

30. Gao J, Zheng P, Jia Y, Chen H, Mao Y, Chen S, et al. Mental Health Problems and Social Media Exposure During COVID-19 Outbreak. Plos one (2020) 15 (4):e0231924.

31. Lawfare. (2020). [Available from: https://www.lawfareblog.com/covid-19and-social-media-content-moderation.

32. Healthcare IT News. (2020). [Available from: https://www.healthcareitnews. com/news/europe/nhs-joins-forces-tech-firms-stop-spread-covid-19misinformation.

33. Medscape. . https://www.medscape.com/viewarticle/930360. [Available from: https://www.medscape.com/viewarticle/930360.

34. Politico. (2020). [Available from: https://www.politico.com/news/2020/03/12/ social-media-giants-are-fighting-coronavirus-fake-news-its-still-spreadinglike-wildfire-127038.

35. Vosoughi S, Roy D, Aral S. The spread of true and false news online. Science (2018) 359(6380):1146-51. doi: 10.1126/science.aap9559

36. Nielsen RK, Fletcher R, Newman N, Brennen JS, Howard PN. Navigating the 'Infodemic': How People in Six Countries Access and Rate News and Information about Coronavirus. In: Misinformation, science, and media. The Reuters Institute for the Study of Journalism and University of Oxford (2020). p. 2020-04.

37. American Psychiatric Association Annual Meeting. Social media and psychiatry: challenges and opportunities. San Francisco, CA (2019). [press release]. https://www.psychcongress.com/.

38. Hall AK, Bernhardt JM, Dodd V, Vollrath MW. The digital health divide: evaluating online health information access and use among older adults. Health Educ Behav (2015) 42(2):202-9. doi: 10.1177/1090198114547815

39. In Time of Social Distancing, Report's Call for Health Care System to Address Isolation and Loneliness Among Seniors Resonates. JAMA Health Forum (2020) 1(3):e200342-e200342. American Medical Association.

40. Santini ZI, Jose PE, Cornwell EY, Koyanagi A, Nielsen L, Hinrichsen C, et al. Social disconnectedness, perceived isolation, and symptoms of depression and anxiety among older Americans (NSHAP): a longitudinal mediation analysis. Lancet Public Health (2020) 5(1):e62-70. doi: 10.1016/S2468-2667 (19)30230-0

41. Perissinotto CM, Cenzer IS, Covinsky KE. Loneliness in older persons: a predictor of functional decline and death. Arch Internal Med (2012) 172 (14):1078-84. doi: 10.1001/archinternmed.2012.1993

42. Moussa Y, Mahdanian AA, Yu C, Segal M, Looper KJ, Vahia IV, et al. Mobile health technology in late-life mental illness: a focused literature review. Am J Geriatr Psychiatry (2017) 25(8):865-72. doi: 10.1016/j.jagp.2017.04.003

43. Schreurs K, Quan-Haase A, Martin K. Problematizing the digital literacy paradox in the context of older adults' ICT use: Aging, media discourse, and self-determination. Can J Commun (2017) 42(2):1-34. doi: 10.22230/ cjc. $2017 \mathrm{v} 42 \mathrm{n} 2 \mathrm{a} 3130$

44. Schueller SM, Hunter JF, Figueroa C, Aguilera A. Use of Digital Mental Health for Marginalized and Underserved Populations. Curr Treat Options Psychiatry (2019) 6(3):243-55.

45. Sarkar U, Gourley GI, Lyles CR, Tieu L, Clarity C, Newmark L, et al. Usability of commercially available mobile applications for diverse patients. J Gen Internal Med (2016) 31(12):1417-26. doi: 10.1007/s11606-016-3771-6 
46. Van Winkle B, Carpenter N, Moscucci M. Why Aren't Our Digital Solutions Working for Everyone? AMA J Ethics (2017) 19(11):1116-24. doi: 10.1001/ journalofethics.2017.19.11.stas2-1711

47. Berkman ND, Sheridan SL, Donahue KE, Halpern DJ, Crotty K. Low health literacy and health outcomes: an updated systematic review. Ann Intern Med (2011) 155(2):97-107. doi: 10.7326/0003-4819-155-2201107190-0005

48. De Barros AC, Leitão R, Ribeiro J. Design and evaluation of a mobile user interface for older adults: navigation, interaction and visual design recommendations. Proc Comput Sci (2014) 27(27):369-78. doi: 10.1016/ j.procs.2014.02.041

49. Grossman LV, Masterson Creber RM, Benda NC, Wright D, Vawdrey DK, Ancker JS. Interventions to increase patient portal use in vulnerable populations: a systematic review. J Am Med Inf Assoc (2019) 26(8-9):85570. doi: 10.1093/jamia/ocz023
50. Alami H, Gagnon M-P, Ahmed MAA, Fortin J-P. Digital health: Cybersecurity is a value creation lever, not only a source of expenditure. Health Policy Technol (2019) 8(4):319-21. doi: 10.1016/j.hlpt.2019.09.002

Conflict of Interest: The authors declare that the research was conducted in the absence of any commercial or financial relationships that could be construed as a potential conflict of interest.

Copyright (c) 2020 Figueroa and Aguilera. This is an open-access article distributed under the terms of the Creative Commons Attribution License (CC BY). The use, distribution or reproduction in other forums is permitted, provided the original author(s) and the copyright owner(s) are credited and that the original publication in this journal is cited, in accordance with accepted academic practice. No use, distribution or reproduction is permitted which does not comply with these terms. 